\title{
Existence of Short Proofs for Nondivisibility of Sparse Polynomials Under the Extended Riemann Hypothesis
}

\author{
Dima Yu. Grigoriev \\ Max Planck Institute of Mathematics \\ 5300 Bonn 1 \\ Marek Karpinski * \\ Dept. of Computer Science \\ University of Bonn \\ 5300 Bonn 1 \\ and \\ International Computer Science Institute \\ Berkeley, California \\ Andrew M. Odlyzko \\ AT\&T Bell Laboratories \\ Murray Hill, NJ 07974
}

\begin{abstract}
We display an existence of the short (polynomial size) proofs for nondivisibility of two sparse multivariate polynomials under the Extended Riemann Hyphothesis (ERH). The divisibility problem is closely related to the rational interpolation problem (whose complexity bounds were determined in [GKS 90] and [GK 91]). In this setting we assume that a rational function is given by a black box (see e.g. [KT 88, GKS 90, K 89]) for eva]uating it.

We prove also that, surprisingly, the problem of deciding whether a rational function given by a black box equals a polynomial belongs to the parallel class NC (see e.g. [KR 90]), provided we know the degree of some sparse representation of it.
\end{abstract}

\footnotetext{
*Supported in part by the Leibniz Center for Research in Computer Science, by the DFG Grant KA 673/4-1 and by the SERC Grant GR-E 68297

Permission to copy without fee all or part of this material is granted provided that the copies are not made or distributed for direct commercial advantage, the ACM copyright notice and the title of the publication and its date appear, and notice is given that copying is by permission of the Association for Computing Machinery. To copy otherwise, or to republish, requires a fee and/or specific permission.

ISSAC' $92-7 / 92 / C A$, USA

- 1992 ACM 0.89791-490-2/92/0007/0117...\$1.50
}

\section{Introduction}

Symbolic manipulation of sparse polynomials, given by binary lists of exponents and nonzero coefficients, appears to be much more difficult than dealing with polynomials in dense encoding (see e.g. [GKS 90, KT 88, P 77a, P 77b]). The sparse representation of polynomials corresponds to the actual size of arithmetic circuits of depth 2 representing them, and excludes the possibility of exponential padding of the size of the input by the nonzero coefficients. The first results in this direction are due to Plaisted [P 77a, P 77b], who proved, in particular, the NP-completeness of divisibility of a polynomial $x^{n}-1$ by a product of sparse polynomials. On the other hand, essentially nothing nontrivial is known about the complexity of the divisibility problem of two sparse integer polynomials. 
(One can easily prove that it is in PSPACE with the help of [M 86].) Here we prove that the problem of nondivisibility of two sparse multivariable polynomials has (polynomially) short proofs (is in NP), provided that the Extended Riemann Hypothesis (ERH) holds (see e.g. [LO 77]).

We prove also that the problem of deciding whether a rational function given by a black bo $x$ equals a polynomial belongs to the parallel class $\mathrm{NC}$, provided we know the degree of some sparse rational representation of it.

\section{Nondivisibility problem for sparse polynomials}

We start with the formulation of the problem. Let $f=\sum_{1 \leq i \leq t} a_{\imath} X^{J_{2}}, g=\sum_{1 \leq i \leq t} b_{i} X^{r K_{1}} \in$ $\mathbb{Z}\left[X_{1}, \ldots, X_{n}\right]$ be two at most $t$-sparse polynomials. Assume that every degree $\operatorname{deg}_{x_{j}}(f)$, $\operatorname{deg}_{x_{j}}(g)<d, 1 \leq j \leq n$ and the bit-size $l\left(a_{i}\right)$, $l\left(b_{i}\right)$ of each integer coefficient $a_{i}, b_{i}$ is less than $M$. The problem is to test, whether $g$ divides $f$. Observe that the bit-size of input data is $O(t(M+n \log d))$.

First, we consider the case $n=1$ of onevariable polynomials $f=\sum_{1 \leq \imath \leq t} a_{\imath} x^{j_{1}}, g=$ $\sum_{1 \leq i \leq t} b_{i} x^{k_{i}}$.

Lemma 1. Any nonzero root of $g$ (also of $f$ ) has multiplicity less than $t$.

Proof. Assume the contrary and let $x_{0} \neq 0$ be a root of $g$ with multiplicity at least $t$. Then $g\left(x_{0}\right)=g^{(1)}\left(x_{0}\right)=\cdots=g^{(t-1)}\left(x_{0}\right)=0$. Hence the $t \times t$ matrix

$$
\begin{array}{rll}
1 & \cdots & 1 \\
k_{1} & \cdots & k_{t} \\
k_{1}\left(k_{1}-1\right) & \cdots & k_{t}\left(k_{t}-1\right) \\
k_{1}\left(k_{1}-1\right)\left(k_{1}-2\right) & \cdots & k_{t}\left(k_{t}-1\right)\left(k_{t}-2\right) \\
\vdots & & \\
k_{1}\left(k_{1}-1\right) \cdots\left(k_{1}-t+2\right) & \cdots & k_{t}\left(k_{t}-1\right) \cdots\left(k_{t}-t+2\right)
\end{array}
$$

is singular. This leads to a contradiction since this matrix by elementary transformations of its rows can be reduced to a Vandermonde matrix $\left(\begin{array}{cccc}1 & 1 & \ldots & 1 \\ k_{1} & k_{2} & \ldots & k_{t} \\ \vdots & & & \vdots \\ k_{1}^{t-1} & k_{2}^{t-1} & \ldots & k_{t}^{t-1}\end{array}\right)$.

Assume that $g$ does not divide $f$. Then there exists a factor $h \in \mathbb{Z}[x]$ of $g$ that is irreducible over $\mathbb{Q}$, and such that its multiplicity $m_{g}$ in $g$ is larger than its multiplicity $m_{f}$ in $f$. The Lemma 1 above shows $m_{g}<t$.

There exist polynomials $u, v \in \mathbb{Q}[x]$ with $\operatorname{deg}(u), \operatorname{deg}(v)<d$ such that $1=u h+v\left(\frac{f}{h^{m_{f}}}\right)$. Taking into account the bounds $l(h), l\left(\frac{f}{h^{m_{f}}}\right) \leq$ $M+d$ that apply to factors of $g, f$, respectively, we obtain $l(u), l(v) \leq M d^{O(1)}$ by virtue of the bounds on the bit-size of minors of the Sylvester matrix (see e.g. [CG 82, L 82, M 82]). Let us rewrite the equality in the following way: $w_{0}=u_{0} h+v_{0}\left(\frac{f}{h^{m_{f}}}\right)$, where $w_{0} \in \mathbb{Z}, u_{0}$, $v_{0} \in \mathbb{Z}[x]$. There exist at most $M \cdot d^{O(1)}$ primes which divide $w_{0}$. Therefore, there exists a prime $p \leq N=(M d)^{O(1)}$ which does not divide any of $w_{0}$, the leading coefficient $l c(g)$ of $g$ and the discriminant of $h$, and moreover the polynomial $h(\bmod p) \in \mathrm{GF}(p)[x]$ has a root in $\mathrm{GF}(p)$ (provided the ERH holds, see [LO 77], Corollary 1.2 on p. 413 or [W 84], the Theorem on p. 182). Then the multiplicity of this root in $f$ equals $m_{f}$ and in $g$ is at least $m_{g}$. 
The nondeterministic procedure under construction guesses a prime $p \leq N$ and an element $\alpha \in \mathrm{GF}(p)$ and tests whether for some $0 \leq i \leq$ $t-1$ one has $g(\alpha)=g^{(1)}(\alpha)=\cdots=g^{(i)}(\alpha)=0$, $f^{(i)}(\alpha) \neq 0, l c(g) \neq 0$ in $\operatorname{GF}(p)$.

One can easily see that if such $p, \alpha$ exist then $g$ does not divide $f$. Indeed, in the opposite case, $(l c(g))^{s} f=g e$ for some integer $s$ and a polynomial $e \in \mathbb{Z}[x]$. Reducing this equation $\bmod p$, one gets a contradiction.

Now we return to the multivariable case. Suppose again that $g$ does not divide $f$. Let $h \in \mathbb{Z}\left[X_{1}, \ldots, X_{n}\right]$ have a similar property to the $h$ in the univariate case. Assume without loss of generality that a variable $X_{1}$ occurs in $h$. Then $g$ also does not divide $f$ in the ring $\mathbb{Q}\left(X_{2}, \ldots, X_{n}\right)\left[X_{1}\right]$ by the Gauss lemma. Consider division of $f$ by $g$ with remainder in the latter ring: $f=g \mu+\theta$. Then $\operatorname{deg}_{X_{\imath}}(\mu), \operatorname{deg}_{X_{i}}(\theta)<d^{2}, 2 \leq i \leq n$ (cf. [L 82]) and the denominators of $\mu, \theta$ are the powers of $l c_{X_{1}}(g) \in \mathbb{Z}\left[X_{2}, \ldots X_{n}\right]$. Hence for some integers $0 \leq x_{2}, \ldots, x_{n} \leq d^{2}+d$ we have $\left(l c_{X_{1}}(g) \cdot l c_{X_{1}}(\theta)\right)\left(x_{2}, \ldots, x_{n}\right) \neq 0$. Therefore, the polynomial $g\left(X_{1}, x_{2}, \ldots, x_{n}\right) \in Z Z\left[X_{1}\right]$ does not divide $f\left(X_{1}, x_{2}, \ldots, x_{n}\right) \in \mathbb{Z}\left[X_{1}\right]$ in the $\operatorname{ring} \mathbb{Q}\left[X_{1}\right]$.

The nondeterministic procedure guesses an index $1 \leq i \leq n$, thus $X_{i}$ (in our argument above its role was played by $X_{1}$ ), the integers $0 \leq x_{2}, \ldots, x_{n} \leq d^{2}+d$ and applies the nondeterministic procedure described before to one-variable polynomials $g\left(X_{1}, x_{2}, \ldots, x_{n}\right)$, $f\left(X_{1}, x_{2}, \ldots, x_{n}\right)$. Thus, we have proved the following

PROPOSITION 1. Nondivisibility of sparse multivariable polynomials belongs to NP provided Extended Riemann Hypothesis holds.

\section{Divisibility problem for sparse rational function given by a black-box}

The proposition 1 can be improved if $t$-sparse $f, g \in \mathbb{Z}\left[X_{1}, \ldots, X_{n}\right]$ are not explicitly given, but we only have a black box (see e.g. [GK 91, GKS 90]) for the rational function $f / g$ provided that $l c_{X_{1}}(g)=1$ and a bound on $d$ is given. This is due to the fact that in the one-variable case we need only a bound on $M$ which one can get even in parallel class $N C$ (cf. [KR 90]) from a black-box relying on the construction from [GK 91] of a big enough number. To do this we proceed as follows.

Assume that $f=\sum_{1 \leq i \leq t_{1}} a_{i} x^{j_{2}}, g=\sum_{1 \leq i \leq t_{2}} b_{i} x^{k_{2}}$, $t_{1}, t_{2} \leq t$ and $g$ has a minimal possible degree for any $t$-sparse representation of the rational function $q=f / g$.

Let $M=\max _{i}\left\{l\left(a_{i}\right), l\left(b_{i}\right)\right\}+1$.

Take successive primes $p_{1}, \cdots, p_{t}$ and for each $p$ among them calculate (by black-box) $q(p), q\left(p^{2}\right), \cdots, q\left(p^{2 t^{2}+1}\right)$. For at least one $p$ all these values are defined, i.e. $g$ does not vanish in these points. Let us fix such $p$.

Lemma 2. At least one of $q(p), q\left(p^{2}\right), \cdots, q\left(p^{2 t^{2}+1}\right)$ has an absolute value greater than $2^{M / 2 t} / t^{4 d t^{2}}$.

Proof. Denote $\mathcal{N}=\max \{|q(p)|, \cdots$, $\left.\left|q\left(p^{2 t^{2}+1}\right)\right|\right\}$. The homogeneous linear system in the indeterminates $A_{i}, B_{i}$

$\sum_{1 \leq i \leq t_{1}} A_{i} p^{s \jmath_{\imath}}=\left(\sum_{1 \leq \imath \leq t_{2}} B_{i} p^{s k_{\imath}}\right) q\left(p^{s}\right), \quad 1 \leq s \leq 2 t^{2}+1$ 
has a unique solution since the polynomials $f, g$ provide a minimal $t$-sparse representation of $q$, hence $\left(\sum_{1 \leq r \leq t_{1}} A_{i} x^{j_{4}}\right) /\left(\sum_{1 \leq i \leq t_{2}} B_{i} x^{k_{1}}\right)=$ $q(x)$. Therefore, each $a_{i}, b_{i}$ equals to a quotient of a suitable pair of $\left(t_{1}+t_{2}-1\right) \times\left(t_{1}+\right.$ $\left.t_{2}-1\right)$ minors of this linear system. Then $\max \left\{\left|a_{i}\right|,\left|b_{i}\right|\right\} \leq\left(\mathcal{N} p^{2 t^{2} d \dot{2} t}\right)^{2 t} \leq\left(\mathcal{N} t^{4 d t^{2}}\right)^{2 t}$. The lemma is proved.

One can construct in $N C$ the integer $t^{4 d t^{2}}$ ([BCH 86]), then by Lemma 2 an integer larger than $2^{M / 2 t}$ and again using [BCH 86] an integer larger than $2^{M}$.

Then the algorithm constructs an integer $N_{0}>36 \cdot 2^{3 M} \cdot d^{5}$. Finally, the algorithm yields the number $N=q\left(q\left(N_{0}\right)\right)$. We claim that $N$ is big enough (see [GK 91]), namely, divide with the remainder $f=e g+\operatorname{rem}(f, g)$, then for each integer $N_{1} \geq N$ we have $0<\left|\frac{\text { rem }(f, g)}{g}\left(N_{1}\right)\right|<\frac{1}{2}$, provided that $\operatorname{rem}(f, g) \neq 0$.

Let us prove the claim. Denote $d_{1}=\operatorname{deg}(f)$, $d_{0}=\operatorname{deg}(g)$. W.1.o.g. assume that $l c(f)>$ 0 . Then $f\left(N_{0}\right)>N_{0}^{d_{1}}-d N_{0}^{d_{1}-1} 2^{M}>\frac{1}{2} N_{0}^{d_{1}}$, $0<g\left(N_{0}\right)<N_{0}^{d_{0}}+d N_{0}^{d_{o}-1} 2^{M}<\frac{3}{2} N_{0}^{d_{0}}$, hence $q\left(N_{0}\right)>\frac{1}{3} N_{0}^{d_{1}-d_{0}}$. On the other hand $f\left(N_{0}\right)<$ $2^{M} d N_{0}^{d_{1}}, g\left(N_{0}\right)>N_{0}^{d_{0}}-2^{M} d N_{0}^{d_{0}-1}>\frac{1}{2} N_{0}^{d_{0}}$, therefore $q\left(N_{0}\right)<2^{M+1} d N_{0}^{d_{1}-d_{0}}$. We get that $q\left(N_{0}\right)<\frac{1}{3} N_{0}$ iff $d_{1}=d_{0}$. In this case $g$ divides $f$ if and only if $f / g \equiv$ const, arguing as in the proof of Lemma 2 the latter identity is equivalent to the equalities $q(p)=\cdots=q\left(p^{2 t^{2}+1}\right)$. So, we assume now that $d_{1}-d_{0}>0$. Notice that the absolute value of each coefficient of $\operatorname{rem}(f, g)$ is at most $\left(\left(d_{1}-d_{0}+2\right) 2^{M}\right)^{d_{1}-d_{0}+2}$ (see e.g. [L 82]). In a similar way $N=$ $q\left(q\left(N_{0}\right)\right)>\frac{1}{3}\left(q\left(N_{0}\right)\right)^{d_{1}-d_{0}}>3^{d_{0}-d_{1}-1} N_{0}^{\left(d_{1}-d_{0}\right)^{2}}$ and $g(N)>N^{d_{0}}-2^{M} d_{0} N^{d_{0}-1}>\frac{1}{2} N^{d_{0}}$. Hence $0<|r e m(f, g)(N)|<\left(\left(d_{1}-d_{0}+\right.\right.$
2) $\left.2^{M}\right)^{d_{1}-d_{0}+2} d_{0} N^{d_{0}-1}<\frac{1}{4} N^{d_{0}}$. This proves the claim.

So, divisibility $g \mid f$ is equivalent to $(f / g)(N)$ being an integer. The number of arithmetic operations of the exhibited algorithm is at most $(t \log d)^{O(1)}$ with the depth $O(\log t \log \log d)$. Thus, the divisibility problem for one-variable rational function given by a black-box, is in NC.

In the multivariable case divide with the remainder $f=e g+\operatorname{rem}(f, g)$ w.r.t. the variable $X_{1}$, namely in the ring $\mathbb{Q}\left(X_{2}, \cdots, X_{n}\right)\left[X_{1}\right]$, thus $e, \operatorname{rem}(f, g) \in \mathbb{Q}\left[X_{1}, \cdots, X_{n}\right]$ since $l c_{X_{1}}(g)=$ 1. After substituting $X_{1}=X^{d^{n-1}}, X_{2}=$ $X^{d^{n-2}}, \cdots, X_{n}=X^{d^{0}}$, we get an equality $\bar{f}=\bar{e} \bar{g}+\overline{r e m(f, g)}$ for nonvanishing identically polynomials $\bar{f}, \bar{e}, \bar{g}, \overline{r e m(f, g)} \in \mathbb{Q}[X]$ and an inequality $\operatorname{deg}_{X}(\bar{g})=d^{n-1} \operatorname{deg}_{X_{1}}(g)>$ $\operatorname{deg}_{X} \overline{\operatorname{rem}(f, g)}$. Therefore $0 \neq \overline{\operatorname{rem}(f, g)}=$ $\operatorname{rem}(\bar{f}, \bar{g})$ and we conclude that $g$ divides $f$ iff $\bar{g}$ divides $\bar{f}$. So, we apply the divisibility test for one-variable case exhibited above to the rational function $\bar{q}=\bar{f} / \bar{g}$.

Hence the number of arithmetic operations can be bounded by $(t n \log d)^{O(1)}$ with the depth $O(\log (t n) \log \log d)$ invoking the bounds for one-variable case.

PROPOSITION 2. The problem of testing whether a sparse multivariable rational function given by a black-box, equals to a polynomial, belongs to NC, provided that a bound on the degree of some $t$-sparse representation $f / g$ is given such that $l c_{X_{1}}(g)=1$. 


\section{Further Research}

There remains a fundamental open problem in symbolic manipulation of polynomials whether the explicit sparse divisibility problem can be solved in polynomial (deterministic or randomized) time. At present we do not know even whether the problem is in NP $\cap$ co-NP (and this even under the assumption of the ERH).

Acknowledgements. The authors thank M. Singer for the number of interesting discussions.

\section{References}

[BCH 86] Beame, P. W., Cook, S. A., Hoover, H. J., LOG Depth Circuit for Division and Related Problems, SIAM J. Comput. 15 (1986), pp. 994-1003.

[CG 82] A. L. Chistov and D. Yu. Grigoriev, Polynomial-time factoring multivariable polynomials over a global field, Preprint LOMI, E-5-82, Leningrad, 1982.

[GK 91] D. Yu Grigoriev and M. Karpinski, Algorithms for sparse rational interpolation, Proc. 1991 ISSAC, pp. 7-13.

[GKS 90] D. Yu. Grigoriev, M. Karpinski, and M. Singer, Interpolation of sparse rational functions without knowing bounds on exponents, Proc. 31 FOCS, IEEE, 1990, pp. 840-846.

[K 89] Karpinski, M., Boolean Circuit Complexity of Algebraic Interpolation Prablems, Technical Report TR-89-027, International Computer Science Institute, Berkeley (1989); in Proc. CSL'88 Lecture Notes in Computer Science, vol. 385 (1989), pp. 138-147.

[KT 88] E. Kaltofen and B. Trager, Computing with polynomials given by black-boxes for their evaluation: $G C D$, factorization separation of numerators and denominators, Proc. 29 FOCS, IEEE, 1988, pp. 296-305.

[KR 90] Karp, R., Ramachandran, V., A Survey of Parallel Algorithms for Shared Memory Machınes, Research Report No. UCB/CSD $88 / 407$, University of California, Berkeley (1988); Handbook of Theoretical Computer Science A, MIT Press, 1990, pp. 870-941. 
[LO 77] J. C. Lagarias and A. M. Odlyzko, Effective versions of the Chebotarev density theorem, in Algebraic Number Fields, A. Fröhlich, ed., Academic Press, 1977, pp. 409-464.

[L 82] R. Loos, Generalized polynomaal remainder sequences, in Computer Algebra: Symbolic and Algebraic Computation, B. Buchberger, G. E. Collins, and R. Loos, eds., Springer, 1982, pp. 115-137.

[M 82] M. Mignotte, Some useful bounds, in Computer Algebra: Symbolic and Algebraic Computation, B. Buchberger, G. E. Collins, and R. Loos, eds., Springer, 1982, pp. 259-263.

[M 86] K. Mulmuley, A fast parallel algorithm to compute the rank of a matrix over an arbitrary field, Proc. 18 STOC, ACM, 1986, pp. 338-339.

[P 77a] D. Plaisted, Sparse complex polynomıals and polynomaal reducıbılity, J. Comput. Syst. Sci., 14, 1977, pp. 210-221.

[P 77b] D. Plaisted, New NP-hard and NP-complete polynomial and integer divisibility problems, Proc. 18 FOCS, IEEE, 1977, pp. 241-253.

[W 84] P. J. Weinberger, Finding the Numbers of factors of a polynomial, J. Algorithms 5, 1984, pp. 180-186. 